\title{
Combined Nerve Block and Topical Anesthesia: An Effective Alternate to General Anesthesia in Hybrid Balloon Sinuplasty Procedures
}

\author{
Sarika S Naik ${ }^{1}$, Chaitra Venkategowda ${ }^{2}$, Narasimha Reddy $^{3}$, Sudhir M Naik ${ }^{4}$
}

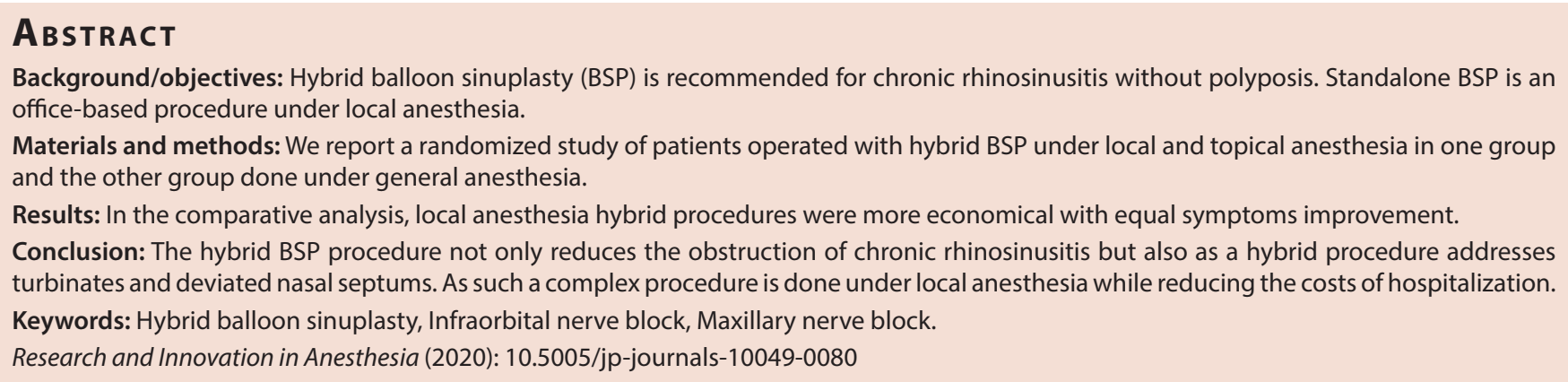

\section{INTRODUCTION}

Balloon sinuplasty (BSP) procedure has similar indications to that of endoscopic endonasal procedures. ${ }^{1}$ The medical therapy is the frontline modality of management of chronic rhinosinusitis but refractory cases may need surgical management. ${ }^{1-3}$ However, BSP has found its role in recurrent acute sinusitis and chronic rhinosinusitis without nasal polyposis. ${ }^{1-3}$ Even though BSP is used to just dilate the sinuses without removing tissues, it has proven to improve the quality of life in patients with chronic rhinosinusitis without nasal polyposis. ${ }^{1-3}$ Hybrid BSP is needed in treating the ethmoidal sinusitis as the endoscopic cold steel clears the ethmoid and the rest of the six sinuses are physiologically dilated. ${ }^{4,5}$ No diagnostic or adjunctive role of BSP is seen in pansinus polyposis, widespread fungal sinusitis, connective tissue disorders at an advanced stage, and malignancy. ${ }^{4,5}$ Also, no role of BSP is documented in sinus headache without positive disease on computed tomography findings. ${ }^{1,4,5}$ As the hybrid BSP procedures need adequate analgesia and anesthesia we have done a comparative study of types of anesthesia used in the similar two groups. One group was operated on with an infraorbital nerve block, maxillary nerve block with topical anesthesia while the other was operated on with conventional general anesthesia.

The study aimed at reducing the overkill effect of functional endoscopic surgery with an alternate BSP procedure. ${ }^{6}$ Balloon sinuplasty gives more physiological clearance as it reduces scarring and biofilm formation when compared with conventional endoscopic surgery. ${ }^{6}$ But addressing the additional features like the inferior turbinates and nasal septum at the same setting needs hybrid procedures under anesthesia. ${ }^{6}$ To reduce the time and costs of general anesthesia, we operated hybrid BSP cases under combined local and topical anesthesia. We compared the advantages of local anesthesia vs general anesthesia in this study which consisted of 25 patients each.
${ }^{1-3}$ Department of Anaesthesia and Critical Care, The Oxford Medical
College Hospital and Research Centre, Bengaluru, Karnataka, India
${ }^{4}$ Department of ENT Head and Neck Oncosurgery, The Oxford Medical
College Hospital and Research Centre, Bengaluru, Karnataka, India

Corresponding Author: Sarika S Naik, Department of Anaesthesia and Critical Care, The Oxford Medical College Hospital and Research Centre, Bengaluru, Karnataka, India, Phone: +91 8088000800, e-mail: bitta301@gmail.com

How to cite this article: Naik SS, Venkategowda C, Reddy N, et al. Combined Nerve Block and Topical Anesthesia: An Effective Alternate to General Anesthesia in Hybrid Balloon Sinuplasty Procedures. Res Inno in Anesth 2020;5(1):6-9.

Source of support: Nil

Conflict of interest: None

\section{Materials and Methods}

We report a randomized study comparing the types of anesthesia used in hybrid BSP cases. Fifty cases of chronic rhinosinusitis not recovering with maximal medical therapy were posted for hybrid BSP surgery. The quantitative computerized scan assessment of patients with Lund Mackay scoring and the qualitative symptom scoring of SNOT 22 laid the criteria for all the 50 patients. The cases were divided into groups I and II and written informed consent was taken for the procedure. The patients in group I were posted for surgery under local and topical anesthesia while group II cases were posted under general anesthesia. Vitals and preoperative assessment were done and all the cases were under the American Society of Anesthesiologists (ASA) grades I and II. In all the cases, nasal decongestion and topical anesthesia $(15 \mathrm{~mL}$ $4 \%$ lidocaine $+2 \mathrm{~mL}$ 1:10,000 adrenaline) were used 1 hour before shifting the cases to the theater (preoperatively). To reduce bias,

(c) The Author(s). 2020 Open Access This article is distributed under the terms of the Creative Commons Attribution 4.0 International License (https://creativecommons. org/licenses/by-nc/4.0/), which permits unrestricted use, distribution, and non-commercial reproduction in any medium, provided you give appropriate credit to the original author(s) and the source, provide a link to the Creative Commons license, and indicate if changes were made. The Creative Commons Public Domain Dedication waiver (http://creativecommons.org/publicdomain/zero/1.0/) applies to the data made available in this article, unless otherwise stated. 
all the nerve block, balloon dilatation, and surgery were done by the same surgeon and anesthesiologist.

In group I, the maxillary block was given first intraorally and later infraorbital nerve block. Two insulin syringes of $1 \mathrm{~mL} 1 \%$ lidocaine were used for infiltration of the maxillary nerve at the greater palatine canal (GPC). $0.2 \mathrm{~mL}$ was infiltrated at the dip of the foramen and later the atraumatic needle was introduced at 45-degree angulation at a horizontal plane to the palate (Fig. 1). The GPC transmits the greater palatine nerve a branch of the pterygopalatine ganglion that carries both general sensory fibers from the maxillary nerve (V2) and parasympathetic fibers from the nerve of the pterygoid canal. With the length of the needle $12.7 \mathrm{~mm}$ going deep rest of the $0.8 \mathrm{~mL}$ of lidocaine was pushed after seeing the negative pressure hemorrhagic aspirate. Infiltration was done on both sides of GPC and later infraorbital nerve block was given bilaterally. Bilateral maxillary block at GPC anesthetizes the entire hemi-maxilla, including teeth, palatal and gingival mucosa, the skin of the midface, maxillary sinus, and nasal cavity.

The infraorbital nerve is blocked by palpating the depression at the infraorbital foramen at $2.5 \mathrm{~cm}$ from the midline on an imaginary line passing from the supraorbital notch, the pupil of the eye, and the second bicuspid tooth (Fig. 2). Three milliliters of $0.25 \%$ bupivacaine and 1\% lignocaine with adrenaline 1: 400,000 for the infraorbital nerve were infiltrated at the opening without entering the foramen ( $1.5 \mathrm{~mL}$ on either side). It is a cutaneous branch of the maxillary nerve emerging through the infraorbital foramen and supplies the skin of the nose, nasal septum, the upper lip and its mucosa, and the lower eyelid and its conjunctiva.

Decongestion in the cavities with $4 \%$ lidocaine $15 \mathrm{~mL}$ in $2 \mathrm{~mL}$ of adrenaline with cotton packs provided topical anesthesia to the turbinates and posterior part of the nasal septum supplied by the sensory branches of the pterygopalatine nerves. The nasal pack in group II was removed and general anesthesia was given. General anesthesia was given with glycopyrrolate, midazolam, and fentanyl as premedication. All the patients were induced with $2-3 \mathrm{mg} / \mathrm{kg}$ of propofol and vecuronium. General anesthesia was maintained with vecuronium and isoflurane. Isoflurane was adjusted to maintain hemodynamic stability with a clear surgical field to operate. Patients were extubated when they were fully awake with all the reflexes intact. Patients were monitored postoperatively for any complications.

Repacking for 3 minutes was done after induction in group II and after the blocks in group I under endoscopy. Balloon dilation was done for maxillary, frontal, and sphenoid sequentially on both sides in all the cases. The inferior turbinate was infiltrated in both
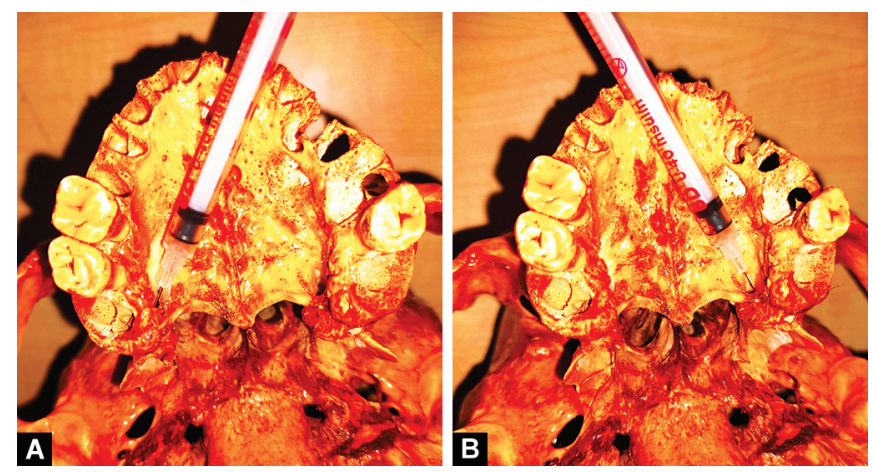

Figs $1 \mathrm{~A}$ and $\mathrm{B}$ : Maxillary nerve block bilaterally at the greater palatine canal nostrils ( $1 \%$ lidocaine + adrenaline) and the turbinate coablation and used to shrink the turbinates. Minimal spurectomy or septal repair was done in all cases wherever necessary.

The procedure was standardized in both the groups with balloon dilatation of all the sinuses frontal, maxillary and sphenoids on either side with minimal cold steel instrumentation like removal of loose lying mucosa, bony spicules, and atraumatic suctioning of the sinuses. The inferior turbinates were coablated wherever necessary and deviations and spurs were addressed in symptomatic cases.

\section{Results}

Four parameters were assessed in groups I and II, (a) time gap interval between shifting the patient to the theater to starting of the procedure, (b) subjective patient analysis score postoperatively, (c) objective assessment of intraoperative hemorrhage and surgeon's operative comfort. No pediatric cases were included and the age group ranged from 18 to 49 years with the average at 37.4 years. In group I, 18 ASA 1 and 17 ASA 2 while in group II 12 ASA grade I and 13 ASA grade II patients seen.

The time gap interval between shifting the patient to the theater to starting of the procedure was 23 minutes in group I while it was 43 minutes in group II. Dilatation of 6 sinuses needed 46 minutes on an average in group I while 41 minutes in group II. Coablation turbinoplasty bilaterally after infiltration of the turbinates with $2 \mathrm{~mL}$ 1\% lidocaine with 1:40,000 adrenaline took 9 minutes on either side in both the groups. Septoplasty was done in all the cases with infiltration of the septum with $1 \%$ lidocaine with 1:400,000 adrenaline. An average of 22 minutes was taken by septoplasty in both groups. The time of recovery was immediate in group I while an average of 22 minutes of recovery was seen in group II. Most of the patients in group II commented on a painless recovery while most of group I commented on an experience of discomfort during the procedure. Ten to fifteen milliliters of blood loss were seen in all cases in both groups.

Hybrid BSP can be taken up under maxillary and infraorbital block with topical anesthesia. Lidocaine infiltrations can be done for turbinates as well as for the septum for the hybrid procedures. General anesthesia can be avoided in less apprehensive and motivated cases. Patient discomfort was seen in most of the local anesthesia cases while surgeon comfort was better with general anesthesia. Local anesthesia drastically reduced the costs of the procedure and better suited for our economic scenario keeping in mind the consumable costs of the balloon used.

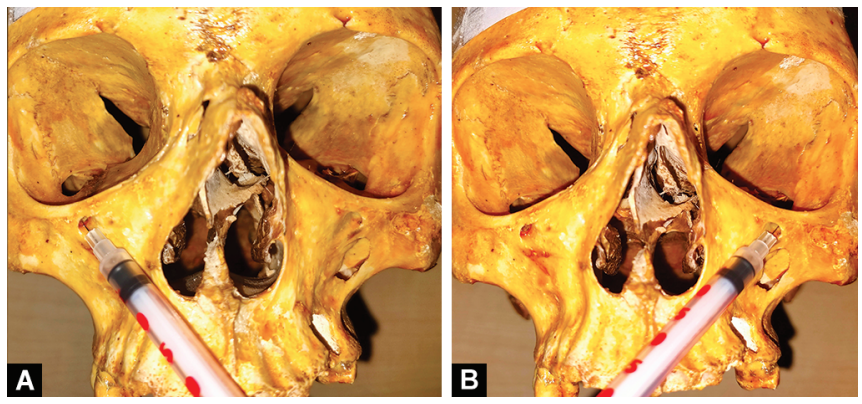

Figs $2 \mathrm{~A}$ and B: Infraorbital nerve block bilaterally at the foramen vertically along the mid-pupillary line 


\section{Discussion}

Chronic rhinosinusitis is an inflammatory condition of the sinonasal mucosa. ${ }^{7}$ Unless polypoidal sinonasal mucosa is obstructing the Ostia of the sinuses most of the cases are managed by maximal medical therapy. ${ }^{7}$ Maximal medical therapy includes analgesics, saline irrigation, intranasal glucocorticoids, oral decongestants, and antibiotics for bacterial infections. ${ }^{7}$ Endoscopic sinus surgeries are being performed for the past three decades and have been overdone in some cases. ${ }^{7}$ So, the paradigm shift is toward the medical line with minimal possible surgical interventions. ${ }^{7}$ Minimal hybrid BSP techniques are aimed at rejuvenating the crucial normal physiological functions of the sinuses. ${ }^{8}$ They aim at maintaining the patency of the ostiomeatal complex, attaining normal mucociliary transport, normal quantity, and quality of secretions. ${ }^{8}$ Endoscopic endonasal surgeries play a major role in treatment of allergic fungal sinusitis, chronic polyposis, mucocele, recurrent sinusitis aggravating pulmonary disease, sinus cysts, tumors, and uncomplicated rhinosinusitis. ${ }^{8}$ The need for lesser intervention in medically refractory chronic rhinosinusitis has paved the way for balloon dilatation of the narrowed openings in the sinuses. ${ }^{9}$ The procedure is similar to endonasal surgeries but rather than doing instrumental corrections of dilatations a small balloon with an illuminated light wire is introduced through the sinus Ostia. ${ }^{9}$ The balloon is hydrodilated for 10 seconds and deflated. ${ }^{9}$ The same procedure is done for all the six sinuses under endoscopic guidance while minimal hybrid procedures like septoplasty, coablation of the inferior turbinates are done wherever necessary. ${ }^{9}$ The regular instruments are used for removal of the loose lying mucosa and bony fragments and also for suctioning the surgical field. ${ }^{9,10}$

Initially developed as an office-based procedure, the scope has not magnified in the present days as the cost of the balloons and the assisting guides are pretty costlier.,10 Also some associated pathologies like inferior turbinates and deviated septums need a hybrid approach to reduce the symptoms. ${ }^{9,10}$ The advantages of the balloon procedure include no removal of the tissues and also it is an office-based procedure. ${ }^{9,10}$ But associated septal and turbinate pathology needs a hybrid approach to reduce symptoms. ${ }^{9,10}$ With revision cases also adding on the indications of balloon procedure it diversifies from the earlier limited indications like recalcitrant chronic sinusitis, frontal, maxillary, or sphenoid sinus disease. ${ }^{11}$ Hybrid balloon procedures extend a step further beyond simply enlarging an ostial opening and include removal of inflamed tissue and bone. ${ }^{11}$ Literature search revealed lesser research on hybrid balloon procedures using local anesthesia techniques., ${ }^{9,11}$

Stankiewicz et al. promoted the BSP procedure under local anesthesia and sedation for isolated maxillary and anterior ethmoid sinusitis cases. He advocated the safety of the office procedure. ${ }^{12}$ Nishioka et al. conducted a retrospective study of 25 patients who underwent hybrid BSP procedures in the office setting using the maxillary nerve block local anesthesia regimen modification., ${ }^{4,5}$ Here, all 25 underwent BSP with variable ethmoidal clearance, 5 septoplasties, and 18 inferior turbinoplasties. ${ }^{4,5}$ All the 25 patients had anesthesia and analgesia for the entire maxilla and even for the hybrid procedures and so they advocated maxillary block for office hybrid procedures. ${ }^{4,5}$ Sikand et al. in their single-arm study of 203 patients followed up for a year reported significant improvement in scores with the office-based BSP done with local and topical anesthesia. $^{13}$

Levine et al. in their 1-year follow-up study of 74 patients operated under BSP under local anesthesia office procedure reported good tolerance and better outcome score in chronic rhinosinusitis and recurrent acute rhinosinusitis patients. ${ }^{14}$ Gould et al. in their study of 303 patients who were followed up for 1 year reported BSP under local anesthesia to be safe effective and welltolerated. ${ }^{15}$ Patients reported a significant reduction in symptom score over 1 year and the local anesthesia was well-tolerated. ${ }^{15}$ Cingi et al. analyzed BSP as an option for limited sinus disease without polyposis and its advantage under local anesthesia makes it a viable therapeutic alternative in comorbid patients who cannot tolerate general anesthesia. ${ }^{1}$

Levine et al. found no drastic increase in the number of BSP procedures after its introduction. ${ }^{8,14}$ Also, it is noted that the local anesthesia office procedure is just eating into the share of conventional surgeries rather than an increase in the overall standalone procedures. ${ }^{8,14}$ Koskinen et al. compared intraoperative characteristics and early postoperative outcomes between endoscopic sinus surgery and BSP. ${ }^{16}$ There was no significant difference between operative time and anesthesia methods in general anesthesia cases. ${ }^{17,18}$ While $33 \%$ of cases were done under local anesthesia as office procedures which cut down costs and improved compliance significantly. ${ }^{17,18}$ Bikhazi et al. in their randomized clinical study on the quality of life after endoscopic sinus surgery or BSP observed good improvement with minimal disease chronic rhinosinusitis. ${ }^{19}$ They also commented that the higher costs of the consumables of the balloon can be compensated by the lower costs of the BSP procedure. ${ }^{19}$

\section{Conclusion}

Hybrid BSP procedures for being able to address many of the minimal sinus and nasal obstructive causes under local anesthesia can be preferred over general anesthesia. A hybrid BSP procedure with local and topical anesthesia is a cost-effective alternative for minimal chronic rhinosinusitis without polyposis.

\section{References}

1. Cingi C, Bayar Muluk N, Lee JT. Current indications for balloon sinuplasty. Curr Opin Otolaryngol Head Neck Surg 2019;27(1):7-13. DOI: 10.1097/MOO.0000000000000506.

2. Ahmed J, Pal S, Hopkins C, et al. Functional endoscopic balloon dilation of sinus ostia for chronic rhinosinusitis. Cochrane Database Syst Rev 2011;7:CD008515. DOI: 10.1002/14651858.CD008515. pub2.

3. Batra PS, Ryan MW, Sindwani R, et al. Balloon catheter technology in rhinology: reviewing the evidence. Laryngoscope 2011;121(1):226232. DOI: $10.1002 /$ lary.21114.

4. Nishioka GJ. The maxillary nerve block for in-office hybrid balloon sinus dilation procedures: a preliminary study. Ear Nose Throat J 2017;96(12):E31-E35. DOI: 10.1177/014556131709601207.

5. Nishioka GJ.Modified in-office maxillary balloon sinus dilation for postprocedure sinus monitoring and access. Int Arch Otorhinolaryngol 2018;22(1):68-72. DOI: 10.1055/s-0037-1601562.

6. Mann WJ, Tóth M, Gouveris $\mathrm{H}$, et al. The drainage system of the paranasal sinuses: a review with possible implications for balloon catheter dilation. Am J Rhinol Allergy 2011;25(4):245-248. DOI: 10.2500/ajra.2011.25.3647.

7. Migirov L, Yakirevitch A, Bedrin L, et al. Endoscopic sinus surgery for medial orbital subperiosteal abscess in children. J Otolaryngol Head Neck Surg 2009;38(4):504-508.

8. Levine $H L$, Sertich AP, Hoisington DR, et al. Multicenter registry of balloon catheter sinusotomy outcomes for 1,036 patients. Ann Otol Rhinol Laryngol 2008;117(4):263-270. DOI: 10.1177/000348940811700405. 
9. Sillers MJ, Melroy CT. In-office functional endoscopic sinus surgery for chronic rhinosinusitis utilizing balloon catheter dilation technology. Curr Opin Otolaryngol Head Neck Surg 2013;21(1):17-22. DOI: 10.1097/ MOO.0b013e32835c05e1.

10. Brodner D, Nachlas N, Mock P, et al. Safety and outcomes following hybrid balloon and balloon-only procedures using a multifunction, multisinus balloon dilation tool. Int Forum Allergy Rhinol 2013;3(8):652-658. DOI: 10.1002/alr.21156.

11. Ference $\mathrm{EH}$, Graber M, Conley D, et al. Operative utilization of balloon vs traditional endoscopic sinus surgery. Laryngoscope 2015;125(1):49-56. DOI: 10.1002/lary.24901.

12. Stankiewicz J, Truitt T, Atkins J, et al. Two-year results: transantral balloon dilation of the ethmoid infundibulum. Int Forum Allergy Rhinol 2012;2(3):199-206. DOI: 10.1002/alr.21024.

13. Sikand A, Silvers SL, Pasha R, et al. Office-based balloon sinus dilation: 1-year follow-up of a prospective, multicenter study. Ann Otol Rhinol Laryngol 2015;124(8):630-637. DOI: 10.1177/0003489415573830.

14. Levine SB, Truitt T, Schwartz M, et al. In-office stand-alone balloon dilation of maxillary sinus ostia and ethmoid infundibula in adults with chronic or recurrent acute rhinosinusitis: a prospective, multi- institutional study with-1-year follow-up. Ann Otol Rhinol Laryngol 2013;122(11):665-671.

15. Gould J, Alexander I, Tomkin E, et al. In-office, multisinus balloon dilation: 1-year outcomes from a prospective, multicenter, open label trial. Am J Rhinol Allergy 2014;28(2):156-163. DOI: 10.2500/ ajra.2014.28.4043.

16. Koskinen A, Penttilä M, Salmi TS. Endoscopic sinus surgery might reduce exacerbations and symptoms more than balloon sinuplasty. Am J Rhinol Allergy 2012;26(6):e150-e156. DOI: 10.2500/ ajra.2012.26.3828.

17. Plaza G, Eisenberg G, Montojo J, et al. Balloon dilation of the frontal recess: a randomized clinical trial. Ann Otol Rhinol Laryngol 2011;120(8):511-518. DOI: 10.1177/000348941112000804.

18. Khalid AN, Smith TL, Anderson JC, et al. Fracture of bony lamellae within the frontal recess after balloon catheter dilatation. Am J Rhinol Allergy 2010;24(1):55-59. DOI: 10.2500/ajra.2010.24.3419.

19. Bikhazi N, Light J, Schwartz M, et al. Standalone balloon dilation vs sinus surgery for chronic rhinosinusitis: a prospective, multicenter, randomized, controlled trial with 1-year follow-up. Am J Rhinol Allergy 2014;28(4):323-329. DOI: 10.2500/ajra.2014.28.4064. 